\title{
Usefulness of Stent Implantation for Treatment of Intracranial Atherosclerotic Stenoses
}

\author{
Kuk Seon Kim, MD', Dae Hyun Hwang, MD', Young Hwan Ko, MD', Ik Won Kang, MD', \\ Eil Seong Lee, MD', You Mie Han, MD', Sun Jung Min, MD', In Soo Kim, MD², \\ Choon Woong Hur, MD², Shiyi Lui, MD³, Tong Lin, MD³, Tongfu You, MD, \\ Haibin Shi, MD ${ }^{4}$ Linsun Li, MD
}

Purpose: We evaluated the usefulness of intracranial stent implantation for treating patients with atherosclerotic stenosis and with recurrent, ischemic, neurological symptoms despite having undergone medical therapy.

Materials and Methods: Between March 2004 and April 2010, we attempted intracranial, stent-assisted angioplasty in 77 patients with 85 lesions (anterior circulation 73 cases, posterior circulation 12 cases) and who had ischemic neurological symptoms with more than $50 \%$ major cerebral artery stenosis. We analyzed the results regarding the technical success rate, complication rate, and restenosis rate during the mean 29.4 month follow-up period.

Results: Intracranial stent implantation was successfully performed in 74 cases $(87.1 \%)$. In nine cases among the 11, failed cases, stent implantation failure was due to the tortuosity of the target vessel. One patient experienced middle cerebral artery rupture during the procedure, and we embolized the vessel using a microcoil. Five patients developed cerebral infarction in three weeks after the procedure, three of whom improved using conservative management, although the other, two patients expired. The mean number of residual stenoses decreased from $72.3 \%$ to $14.7 \%$. Three patients demonstrated significant in-stent restenosis, i.e. more than $50 \%$, during the follow-up period.

Conclusion: As stent-assisted angioplasty in intracranial, atherosclerotic stenosis is effective and relatively safe, it can be considered as an alternative treatment for patients with recurrent, ischemic, neurologic symptoms despite having undergone medical therapy.

Key Words : Atherosclerosis, Intracranial; Stents; Angioplasty

\footnotetext{
'Department of Radiology, Hallym University Hangang Sacred Heart Hospital, Seoul, Korea

${ }^{2}$ Department of Neurosurgery, Myungji St. Mary's Hospital, Seoul, Korea

${ }^{3}$ Department of Emergency, Shanghai Saint Luke's Hospital, Shanghai, China

${ }^{4}$ Department of Radiology, Jiangsu province Hospital, Nanjing Medical University, Nanjing, China

Received May 16, 2011; accepted after revision July 24, 2011.

Correspondence to: Dae Hyun Hwang, MD, Department of Radiology, Hangang Sacred Heart Hospital, 94-200 Yeongdeungpo-dong 2-ga, Yeongdeungpo-gu, Seoul 150-719, Korea.

Tel. 82.2.2639.5225 Fax. 82.2.2679.0121 E-mail: mddhhwang@naver.com

This is an Open Access article distributed under the terms of the Creative Commons Attribution Non-Commercial License (http://creativecommons.org/licenses/by-nc/3.0) which permits unrestricted non-commercial use, distribution, and reproduction in any medium, provided the original work is properly cited.
} 
Stroke is the second most important cause of death in Korea followed by cancer and it is the leading cause of physical disability in Koreans [1]. In Asians, the incidence and mortality rate due to stroke is higher than that in the Western population, although the rate of coronary heart disease is lower in Asians. Racial differences have been suggested in the distribution of cerebral atherosclerosis [2].

Little is known about the natural history of stenosis caused by intracranial atherosclerosis. However, as many as $6-29 \%$ of all ischemic strokes are related to stenotic lesions located in large, intracranial vessels, and an increased risk for stroke, heart disease, and death has been consistently observed [3]. In a Warfarin Aspirin Symptomatic Intracranial Disease (WASID) trial, $14 \%$ and $23 \%$ of the patients who had a transient ischemic attack (TIA) or stroke attributed to a highgrade, intracranial stenosis had another ipsilateral ischemic stroke during the following year of that study even though they were on medical therapy [4]. Recently, percutaneous transluminal angioplasty and stenting have been proposed as an alternative treatment for patients with intracranial atherosclerotic stenosis symptoms despite their medical therapy $[5,6]$.

We report here our results for angioplasty/stenting the intracranial arteries of 85 consecutive patients. We have included the early and late clinical outcomes, and we compared our results to those which have already been reported in the literature.

\section{MATERIALS AND METHODS}

Between March 2004 and April 2010, 85 intracranial arteries in 77 patients were treated by means of stentassisted angioplasty at our institutions. The inclusion criteria for stent-assisted angioplasty were recurrent symptoms caused by intracranial artery stenosis while the patient was being treated with the optimal dose of an antiplatelet or anticoagulation medication and angiographically proven, significant atherosclerotic stenoses (more than 50\%) that were responsible for the patients' symptoms. The patient age ranged from 37 to 77 years (mean, 60.8 years). There were 43 males and 42 females. The institutional review board approved this retrospective study and waived informed consent.

All the patients underwent preoperative computed tomography (CT) or magnetic resonance imaging (MRI) or both as well as cerebral digital subtraction angiography. Pre-procedural quantitative angiographic measurements were obtained in both the target vessel and the lesion. The degree of stenosis was calculated according to the NASCET (North American Symptomatic Carotid Endarterectomy Trial) criteria for grading carotid stenosis. All patients were treated electively and were premedicated with aspirin $100 \mathrm{mg}$ and clopidogrel $75 \mathrm{mg}$ for a minimum of three days prior the intervention. All procedures were done under local anesthesia, and during the procedures each patient received 5,000-8,000 IU of intravenous heparin in order to attain an activated clotting time (ACT) of more than 250 seconds. Percutaneous access was obtained via a femoral artery. Access to the stenotic artery was achieved using a $6 \mathrm{~F}$ guiding catheter (Envoy; Cordis Endovascular Corporation, Miami, FL, U.S.A.). The stenotic lesion was crossed using a $0.010-0.014$ inch microguidewire, and the balloon catheter was placed over the microguidewire and then directed across the lesion. We used balloon catheters with diameters ranging from 2 to $3 \mathrm{~mm}$ and with lengths of 10 or 20 $\mathrm{mm}$, according to the target vessel size, and we were careful not to allow any over-dilatation. Balloon inflation was performed slowly and was maintained for 10 to 20 seconds using a disposable inflation device that allowed for exact pressure control. In all, we implanted eight kinds of stents, i.e. 46 Endeavor (Medtronic Vascular, $n=46$ ), Vision (Abbott, $n=14$ ), Flexmaster (Abbott, $n=10$ ), Arthos pico (AMG International, $\mathrm{n}=6$ ), Neuroform (Boston Scientific, $\mathrm{n}=3$ ), Guidant (Abbott, $\mathrm{n}=3$ ), Tsunami (Terumo $\mathrm{n}=2$ ), and Driver (Medtronic Vascular, $\mathrm{n}=1$ ).

Antiplatelet therapy with clopidogrel and aspirin was continued for 4-12 weeks following the treatment, and then aspirin was continued during the remainder of each patient's life. Additional boluses of heparin were given to the patients during the procedure in order to maintain an ACT between 200 and 250 seconds and

Table 1. Summary of 85 Cases of Intracranial Stenting

\begin{tabular}{lc}
\hline Characteristic & Value \\
\hline Patient $(\mathrm{n}=77)$ & 60.8 years \\
Mean age & $43: 42$ \\
Male : Female & \\
Location $(\mathrm{n}=85)$ & 40 \\
ICA & 33 \\
MCA & 8 \\
Basilar artery & 4 \\
Vertebral artery & \\
\hline
\end{tabular}

Abbreviations: ICA, intracranial carotid artery; MCA, middle cerebral artery (10\%) 


\section{Stent Implantation for Intracranial Atherosclerotic Stenoses}

which was not reversed at the end of the procedure.

We evaluated the procedure success rate, the periprocedural complications during the 30-day periprocedural period, and any symptom recurrence or restenosis which occurred during the mean 29.4 month (from 3 to 72 months) follow-up period. We defined procedural success as a final luminal narrowing of less than $30 \%$ of the normal luminal diameter. Angiographic restenosis seen on conventional cerebral angiography was defined as stenosis of more than $50 \%$ of the luminal diameter.

The stenosis rates before and after stent-assisted angioplasty and those seen on follow-up were compared using the paired Student's t-test. A probability of P less than .05 was considered statistically significant. SPSS 17.0 (SPSS-PC Inc., Chicago, IL, U.S.A.) statistical software was used.

\section{RESULTS}

The patient characteristics and lesion locations are summarized in Table 1. The lesion locations were the intracranial internal carotid artery $(\mathrm{ICA})(\mathrm{n}=40)$ (Fig. $1)$, middle cerebral artery $(\mathrm{MCA})(\mathrm{n}=33)$, vertebral
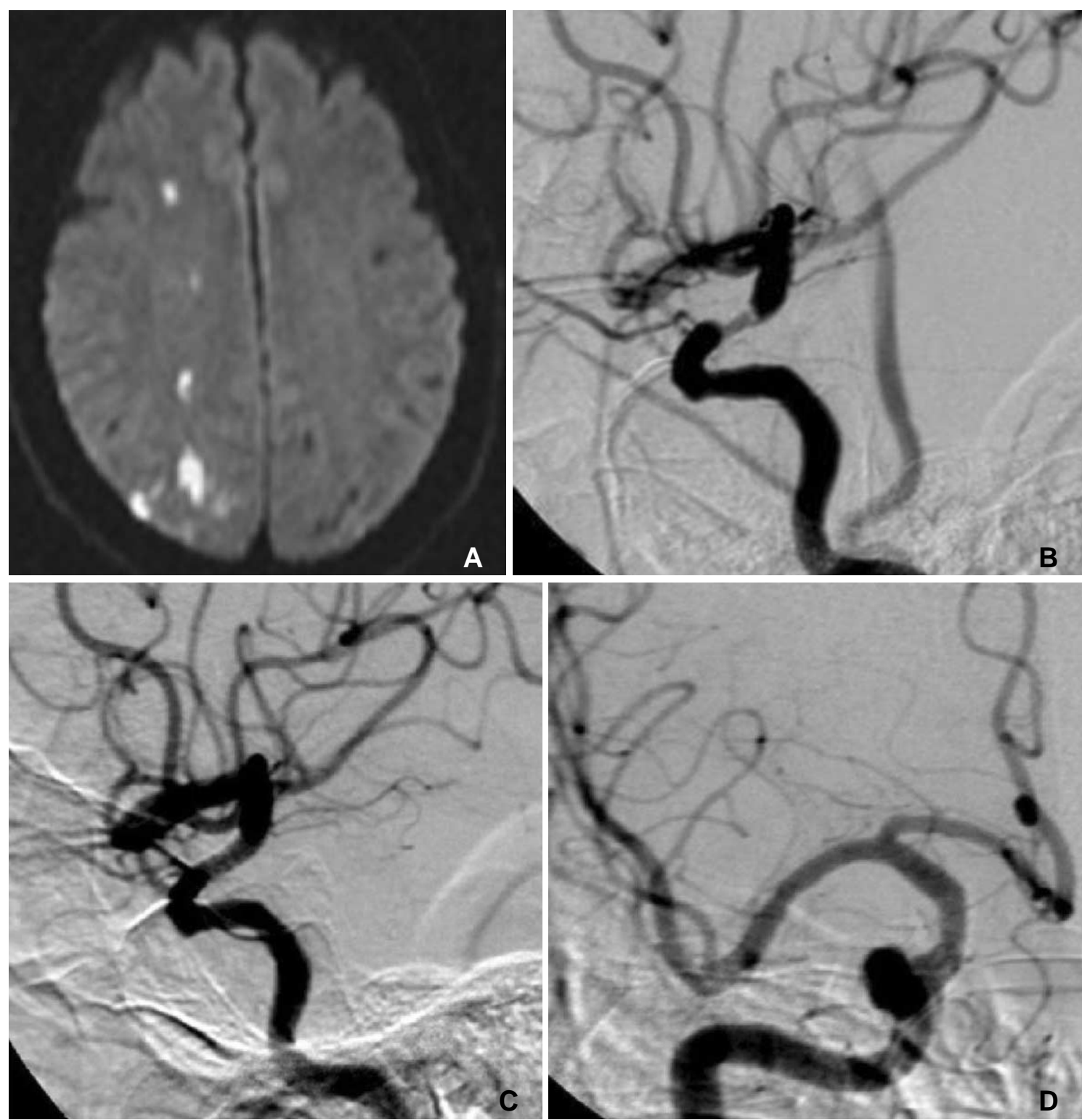

Fig. 1. 62-year-old male who presented left-side hemiparesis and dysarthria.

A. Diffusion-weighted MRI revealed acute infarction in the right parietal lobe.

B. Pre-procedural angiography showed luminal narrowing of the distal right ICA.

C. Post-procedural angiography showed good patency of the flow.

D. Follow-up angiography eight months after the procedure showed no restenosis. 
artery $(n=8)$, and basilar artery $(n=4)$. Available angiograms showed significant improvement of the stenosis, with the mean preprocedural stenosis of the treated lesion being $72.3 \%$ (range, 63-90\%) and the mean postprocedural residual stenosis $14.7 \%$ (range, $0-25 \%)$.

The overall success in reaching the target lesion with stents was $87.1 \%$ (74 of 85 targets), while nine procedures were aborted because of our inability to manipulate the device in the tortuous anatomy of an ICA $(n=8)$ or an aortic arch $(n=1)$. One procedure failed technically because of kinking of the device.
Another procedure failed because insufficient sedation resulted in poor patient cooperation.

In one patient, the MCA was ruptured during the balloon dilatation and the ruptured segment was occluded with microcoils. Follow-up CT scanning showed progressively decreasing subarachnoid hemorrhage and no neurological symptoms.

Table 2 summarizes the clinical and technical results and provides the follow-up data. Nine patients were lost to follow-up, and the data of patients who did not undergo stent implantation were excluded. Therefore, clinical follow-up was available in 65 patients, and the
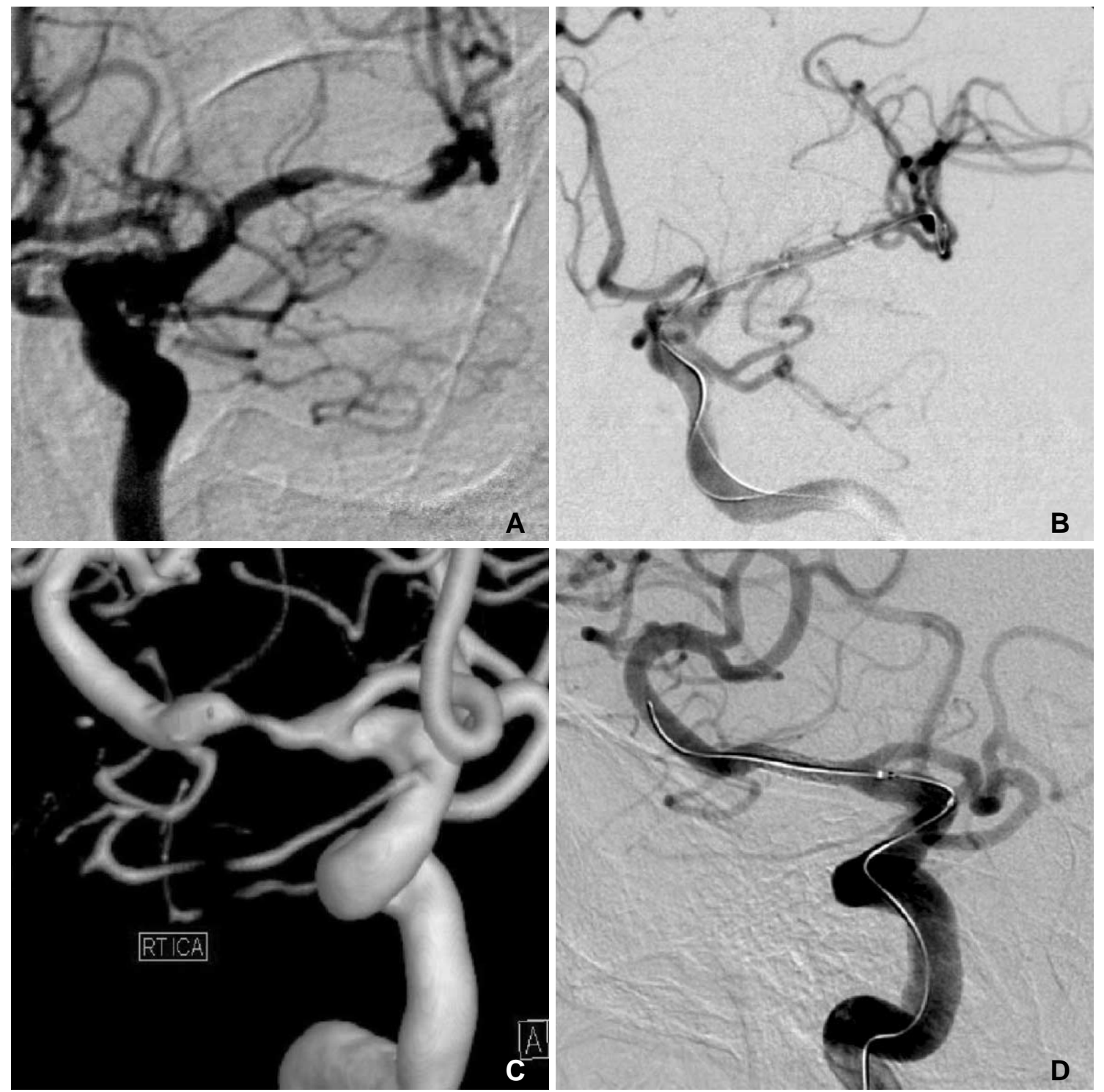

Fig. 2. 69-year-old female who presented right hemiparesis and dysarthria.

A. Angiography showed severe stenosis in the left MCA in the distal M1 portion.

B. After stent-assisted angioplasty, the left MCA was completely recanalized.

C, D. The patient presented with recurrent ischemic neurologic symptoms on the contralateral side six months after the procedure. As her symptoms were related to severe stenosis in the right MCA M1 segment, stent-assisted angioplasty was performed. 

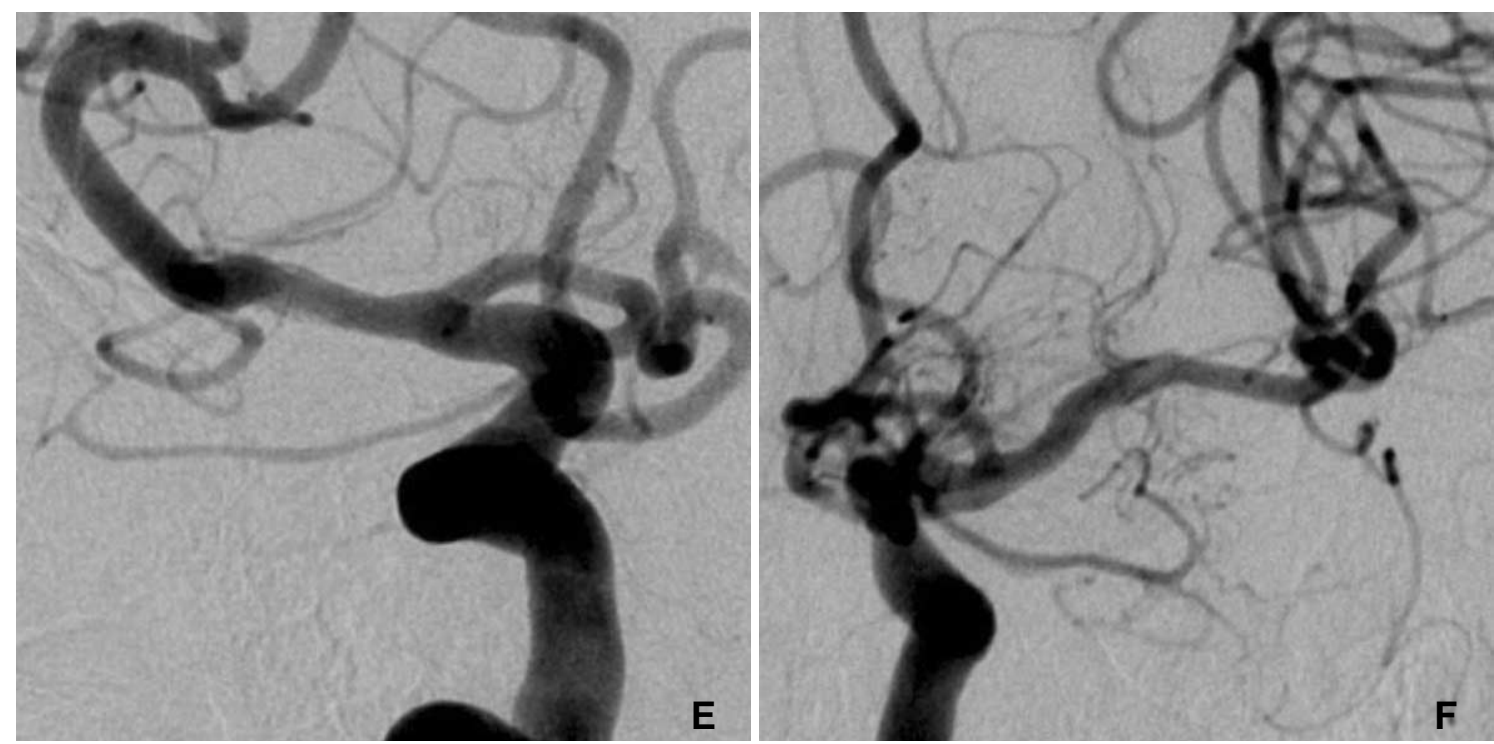

Fig. 2. E, F. Follow-up angiography performed both 13 and 19 months after the procedure, shows good flow patency with stent implantation in the right and left MCA, respectively.

follow-up period ranged from three to 72 months (mean follow-up, 29.4 months). Clinical success was defined as a patient having no recurrent symptoms or angiographic restenosis and was observed in $84.6 \%$ of our patients ( 55 of 65 cases).

During the follow-up period, two patients developed TIA symptoms and all of their MR examinations revealed newly developed ischemic or infarcted lesions associated with the artery we had already treated. The symptoms and lesions improved in both of these patients with the use of heparin and conservative management. Two other patients developed subacute occlusion of the treated arteries (ICA) three weeks after the procedure. Both of these patients expired five weeks after the procedure due to multifocal intracranial hemorrhage. One patient chose to stop taking the anticoagulant drugs, and ischemic neurologic symptoms began to develop two weeks after the procedure, MR imaging revealed a newly developed infarction, and an angiogram showed subacute occlusion of treated artery (MCA). The lesion developed hemorrhagic transformation one week later, and a follow-up CT scan showed progressively improving lesions without neurologic sequalae. One patient died six weeks following the procedure because a slip down resulted in a traumatic intracerebral hematoma, 6 weeks after the procedure.

Conventional angiographic follow-up was available in 31 of the 74 study patients. One patient who presented right hemiparesis and dysarthria had severe stenosis in the left MCA distal M1 portion. Six months
Table 2. Summary of the Procedural and Follow-up Results

\begin{tabular}{lc}
\hline Characteristic & Value \\
\hline Technical results & \\
Technical success rate & $87.1 \%(74 / 85)$ \\
Technical failure rate & $12.9 \%(11 / 85)$ \\
Stenosis & $72.3 \%$ \\
Pre-procedural stenosis & $14.7 \%$ \\
Post-procedural stenosis & \\
Complications & 1 \\
MCA rupture & 5 \\
${ }^{*}$ Ischemic stroke & 3 \\
${ }^{\dagger}$ Death & \\
Follow-up results & 61 \\
No significant change & 4 \\
Re-stenosis & 9 \\
Follow-up loss & \\
\hline
\end{tabular}

TIA: Transient ischemic attack

* Including 2 transient ischemic attack

${ }^{\dagger}$ Two reperfusion injury after subacute infarction and one traumatic intracerebral hematoma

after stent-assisted angioplasty, the ischemic neurologic symptom recurred and angiography showed MCA stenosis of the opposite side. Another stent was then implanted in the right MCA. After 19 months, followup angiography showed no restenosis in either MCA 
stent (Fig. 2). Three patients showed restenosis of the stent-treated artery during the follow-up period. One patient in whom another stent was implanted in the ICA, developed ischemic symptoms three month later. She then underwent superficial temporal artery-middle cerebral artery bypass surgery. In one patient who underwent balloon dilatation, no further restenosis was observed on the follow-up angiogram. Another patient showed restenosis on the follow-up angiogram, but was without symptoms.

\section{DISCUSSION}

The indication for stenting of intracranial vessels depends on both the risks of the untreated or the medically treated disease and the safety and effectiveness of the interventional procedure [7]. The natural history of intracranial stenoses has not been studied as well as that of extracranial stenoses, although intracranial stenoses consistently have a high incidence of stroke. In one study, patients with intracranial ICA stenoses and who were followed for an average of 3.9 years had a TIA or stroke rate of $27.3 \%$ [3].

In the External Carotid/Internal Carotid Artery Bypass Trial [8], the medical patient group, i.e. those treated with aggressive stroke risk factor management and $1300 \mathrm{mg}$ of aspirin daily, had an annual mortality and stroke rate of $8 \%$ to $10 \%$. In the WASID trial [4], the one-year rate of ischemic stroke in the territory of the stenotic artery was approximately $12 \%$ in those patients with symptomatic intracranial stenosis of more than $50 \%$ and who were treated with either aspirin or warfarin. And in the GESICA study [9], the two-year recurrence rate of ischemic events in the territory of the stenotic artery, was $38.2 \%$ despite medical treatment.

Because of continually advancing device technology, intracranial stenting is now feasible. However, the clinical outcome varies from moderate- to high-risk rates for morbidity and mortality. To date, the only prospective, multicenter study that has been conducted is the SSYLVIA study [10] which demonstrated stroke rates of $6.6 \%$ and $13.1 \%$ at 30 days and one year, respectively.

In our clinical experience, stent implantation for intracranial atherosclerotic stenosis had a relatively high success rate $(87.1 \%)$ and few procedure-related complications $(1.2 \%)$, and most of our patients who had a successful procedure experienced good clinical outcomes and were free of ischemic symptoms. Our restenosis rates $(4.0 \%)$ are similar to those of previous reports [11].
In this study, the angiographic follow-up data is limited because clinically symptom-free patients without any ischemic lesion seen on follow-up MRI examination, did not have to undergo follow-up angiography. As we considered patients with a good clinical outcome as those without significant restenosis, this may have affected our low rate of restenosis.

Five patients $(6.5 \%)$ developed subacute occluded infarction or intracranial hemorrhage as following stent-assisted angioplasty of intracranial vessels, there is a high risk for intracranial hemorrhage, particularly within the first hours after the treatment, and due to hyperperfusion of the previously hypoperfused brain parenchyma with lost autoregulation or small vessel injury in recently infarcted tissue. Therefore, patients' blood pressure must be controlled, and large to prevent subacute infarction [11].

Angulation and tortuosity of an affected vessel are the most important factors in failed stent implantation. As they may also be associated with stent rigidity and overcome technical problem, we expect an increasing success rate as we gain more clinical experience and with improvement of the device [13].

Stent-assisted angioplasty for intracranial stenosis is becoming increasingly available for applying in medical refractory stenosis and is being used in patients before other types of surgical therapy. Before the interventional procedure, both a perfusion study and a reserve capacity test must be obtained. Evaluation of the morphology and localization of the stenosis by preprocedural angiography, are also needed. In addition, side branches and perforators closing the stenotic lesion are important informations those should be evaluated before stent implant [14].

As stent-assisted angioplasty used to treat intracranial atherosclerotic stenosis, is both effective and relatively safe, it should be considered as an alternative treatment for patients with recurrent ischemic neurologic symptoms despite having undergone medical therapy. However, large patient cohorts and long-term followup data will be needed in order to provide positive evidence of its considerable benefit. We expect that increased clinical experience and improvement of the device will allow high clinical success rate and a low number of procedural complications.

\section{References}

1. Kim JS. Stroke in Korea. International Congress Series, Proceedings of the 13th International Atherosclerosis Symphosium 2004;1262:348-351

2. Suh DC, Lee SH, Kim KR, Park ST, Lim SM, Kim SJ, et al. Pattern of atherosclerotic carotid stenosis in Korean patients with 


\section{Stent Implantation for Intracranial Atherosclerotic Stenoses}

stroke: different involvement of intracranial versus extracranial vessels. AJNR Am J Neuroradiol 2003;24:239-244

3. Lylyk P, Cohen JE, Ceratto R, Ferrario A, Miranda C. Angioplasty and stent placement in intracranial atherosclerotic stenoses and dissections. AJNR Am J Neuroradiol 2002;23:430436

4. Chimowitz MI, Kokkinos J, Strong J, Brown MB, Levine SR, Silliman S, et al. The Warfarin-aspirin symptomatic intracranial disease study. Neurology 1995;45:1488-1493

5. Kim DJ, Lee BH, Kim DI, Shim WH, Jeon P, Lee TH. Stent assisted angioplasty of symptomatic intracranial vertebrobasilar artery stenosis: feasibility and follow up results. AJNR Am J Neuroradiol 2005;26:1381-1388

6. Suh DC, Kim JK, Choi JW, Choi BS, Pyun HW, Choi YJ, et al. Intracranial stenting of severe symptomatic intracranial stenosis: results of 100 consecutive patients. AJNR Am J Neuroradiol 2008;29:781-785

7. Hahnel S, Ringleb P, Hartmann M. Treatment of intracranial stenoses using the Neuroform stent system: initial experience in five cases. Neuroradiol 2006;48:479-485

8. The EC/IC Bypass Study Group. Failure of extracranial-intracranial arterial bypass to reduce the risk of ischemic stroke. Results of an international randomized trial. N Engl J Med 1985;313:
1191-1200

9. Mazighi M, Tanasescu R, Ducrocq X, Vicaut E, Bracard S, Houdart E, et al. Prospective study of symptomatic atherothrombotic intracranial stenoses: the GESICA study. Neurology 2006;66:1187-1191

10. SSYLVIA Study Investigators. Stenting of symptomatic atherosclerotic lesions in the vertebral or intracranial arteries (SSYLVIA): study results. Stroke 2004;35(6):1388-1392

11. Groschel K, Schnaudigel S, Pilgram SM, Wasser K, Kastrup A. A systematic review on outcome after stenting for intracranial atherosclerosis. Stroke 2009;40:e340-e347

12. Qureshi AI, Saad M, Zaidat OO, Suarez JI, Alexander MJ, Suri FK, et al. Intracerebral hemorrhages associated with neurointerventional procedures using a combination of antithrombotic agents including abciximab. Stroke 2002;33: 1916-1919

13. Choi HW, Koo YB, Lee TH, Kim HJ, Lee JW, Kim CW, et al. New techniques for intracranial stent navigation in patients with tortuous arteries. J Korean Soc Radiol 2005;52:101-106

14. Weber W, Mayer TE, Henkes H, Kis B, Hamann GF, Altedorneburg GS, et al. Stent-angioplasty of intracranial vertebral and basilar artery stenoses in symptomatic patients. EJR Eur J Radiol 2005;55:231-236 